\title{
Sur le divan
}

C'est sous ce titre qu'une pièce de théâtre réaliste a été diffusée pour la première fois le 12 juillet 2006 par la télévision suisse alémanique dans le cadre de l'émission «Rundschau» ${ }^{*}$. Les personnages principaux en sont le célèbre divan du psychothérapeute et deux de ses patientes. D'autres acteurs, tel que le président de la Société de discipline médicale et un porte-parole de l'OFSP ont également eu droit à la parole. Les uns défendaient le mandat confié au psychothérapeute et les autres s'insurgeaient contre les coûts élevés d'une thérapie de longue durée en insistant sur la nécessité d'un meilleur contrôle. Personne n'était convaincant.

La fille anorexique a parlé de ses trois dernières années de thérapie en comparant les photos d'une époque où elle était nourrie au moyen d'une sonde avec celles d'aujourd'hui où son état s'est fortement amélioré. Mais elle a aussi mentionné ses peurs existentielles et idées de suicide persistantes ainsi que la nécessité du suivi médical. Le cas de la mère obèse, filmée avec son mari au bord de l'Aar, là où le cadavre de son fils noyé a été retrouvé, est différent. Elle expliqua que depuis une demi-année, elle se rend, elle aussi, en consultation parce qu'elle pleure pendant des heures et qu'elle a perdu tout contact social. Deux situations très différentes donc, au sujet desquelles les téléspectateurs devaient se faire une opinion. Mais quelle opinion?

Les acteurs improvisés ne suscitent en général pas de grandes émotions. En l'occurrence, leurs commentaires étaient exagérément prosaïques, peut-être en réaction à la lumière inhabituelle jetée sur leur vie intime. «Oui, mon état s'est stabilisé» ou «cela fait du bien d'en parler» ou, concernant l'importance des discussions: «il (le médecin) est simplement un point de contact où l'on se rend, semblable à une pompe à essence où on va faire le plein pour sa voiture». Le psychiatre contribua également à banaliser la situation en expliquant qu'il était un bon auditeur et un être humain à qui l'on pouvait tout confier. Il s'enquiert, en effet, de la promenade avec le chien et des actes quotidiens: «Nous sommes là pour écouter les soucis de nos patients, des choses très ordinaires». Quant à la question de la dépendance: «elle ne devrait pas exister». Il apparaissait ainsi que les gens étaient perdus dans leur solitude et qu'ils avaient besoin d'un interlocuteur à 190 francs l'heure. Ce qui sem- blait évident dans un cas ne l'était plus dans l'autre. L'intervention du président de l'association des psychothérapeutes ne fut pas d'un grand secours, selon laquelle il se sentait discriminé par les nouvelles obligations d'annoncer. Ses deux collègues lui emboîtèrent le pas en signalant l'incompétence des médecins-conseils étrangers à la spécialisation. Ils indiquèrent en outre qu'il n'est pas possible d'élaborer un plan de traitement après six séances déjà car «la plupart du temps, le cas évolue différemment que prévu». Face à cela, le porte-parole de l'OFSP serinait, sous forme de leitmotiv, les schémas habituels relatifs à la baisse des coûts et à une utilisation plus efficace des primes par une meilleure sélection et une qualité améliorée.

Ce fut plus un moment didactique qu'une pièce de théâtre car, selon Neil Postman, c'est le support qui forme le message («the medium is the message»). Le support d'images met davantage l'accent sur l'illustration d'une situation que sur les paroles explicatives. Cette émission l'a malheureusement prouvé, même si la série dont elle fait partie se vante d'être au top de l'actualité et de l'investigation en profondeur. Trop de messages encombraient cette émission trop brève, des liens complexes avaient été simplifiés de manière intolérable et le nombre des questions dépassait celui des réponses. On fit silence sur les solutions thérapeutiques de rechange et les évolutions spontanées et on n'expliqua pas ce qui délimite un deuil normal d'une réaction morbide. Celui qui ne connaît pas l'arrière-plan se trouve insécurisé et perplexe. Une méfiance diffuse se répand, même là où elle ne devrait pas avoir cours; elle est renforcée par les explications d'experts de bonne volonté, lesquels banalisent toutefois les situations et ne sont d'aucune aide pour les profanes. Le bref message de l'OFSP était au moins simple et compréhensible pour tout un chacun, mais guère dans l'intérêt des psychiatres. Ce fut une pièce didactique à laquelle le corps médical ne devrait pas participer. La partie jouée s'est terminée sur un match nul, il n'y eut ni penalty ni arbitre compétent. Les téléspectateurs en bonne santé plaideront pour des contrôles accrus, les personnes malades pour la solidarité. En conclusion, les deux camps feraient bien de s'inspirer du diction indien: «Là où les éléphants marchent, l'herbe souffre.»

Erhard Taverna 